\title{
Biomechanical Analysis of Fatigue Behavior of a Fully Composite-based Designed Hip Resurfacing Prosthesis
}

\author{
K. Chergui ${ }^{1}$, H. Ameddah ${ }^{2 *}$, H. Mazouz ${ }^{1}$ \\ ${ }^{1}$ Research laboratory in production (LRP), University of Batna 2, BATNA 05000, Algeria \\ e-mail: k.chergui@ univ-batna2.dz \\ e-mail: h.mazouz@ univ-batna2.dz \\ ${ }^{2}$ Laboratory of Innovation in Construction, Eco-design, and Seismic Engineering (LICEGS), \\ University of Batna 2, Batna 05000, Algeria \\ e-mail: h.ameddah@univ-batna2.dz \\ *corresponding author
}

\begin{abstract}
The Hip resurfacing prosthesis is subjected to different stresses resulting from the different positions of the human walk, thereby generating dynamic stresses that vary with time, leading the implant material to fatigue failure. It is important to study the fatigue behavior of the prosthesis material and to ensure its long lifetime. We proposed a new composite material named CF/PA12 composed of carbon fibers with a polyamide 12 resin, whose biocompatibility had been demonstrated in laboratories. In this study, we investigated the static and dynamic behavior at different Gait cycle positions of a Hip resurfacing prosthesis entirely made of new CF/PA12 composite. A fatigue behavior will be deducted by a Finite Element Analysis using the commercial SolidWorks software compatible with the Abaqus finite element code. Static and dynamic analysis were conducted considering normal walking and climbing stairs loading at different Gait cycle percentages of 2, 13, 19, 50 and $63 \%$. The results obtained showed that Hip resurfacing prosthesis fully made of new CF/PA12 composite was very far from fatigue and therefore from failure.
\end{abstract}

Keywords: Hip resurfacing prosthesis, fatigue behavior, CF/PA12 composite, FEA

\section{Introduction}

The Hip resurfacing prosthesis is an alternative to the total Hip prosthesis, usually implanted in active and dynamic young patients. The forces applied to the Hip implant due to human activities generate dynamic stresses that vary over time and cause material fatigue and rupture of the implant, so the evaluation of the lifetime of biomaterials and structures in service is essential. The two main implant elements are the head and the cup, which ensure functionality of the joint. Many studies have been carried out in order to study the biomechanics of various activities constituting the daily life of patients to quantify the forces acting at the joints. It has been demonstrated that the joints undergo shocks at each contact of the heel with the sol, generating a force that can be up to one and a half times than the forces usually measured (Hausselle 2007). It has been 
demonstrated that the head and the acetabulum of a Hip prosthesis were subjected to fatigue compression (Hamza 2002). A Large research exists in the literature on fatigue and rupture behavior on total Hip arthroplasty with various materials. Mechanical properties of a composite material proposed by J.U.Perez (2012) named ZTA (zirconia toughened alumina), combined with excellent biocompatibility, resistance to wear and shock and more resistance to stresses before it presents a crack, make this material the best option for total Hip prosthesis. In this field, nacre can be reliably compared with existing medical bioceramics (A12O3 and $\mathrm{ZrO} 2$ ), with the aim of producing implant biomaterials with favorable compression fatigue behavior (Hamza 2016).

For hip resurfacing prosthesis frictional couple, mechanical behavior is the most treated, ceramic on ceramic couple is proposed to avoid the potential problem caused by metal ions generated by current cobalt-chromium prosthesis. Using metallic femoral and acetabular prostheses with alumina coatings instead of whole alumina prostheses was found to significantly reduce the predicted contact pressure distribution (Ahmet C. Cilingir 2010). The study of Bidyut Pal and al. (2010) showed that the use of stiffer ceramic components elevates stresses and strain coupled with increased stress/strain shielding in the resurfaced femur.

On the other hand, the fatigue and rupture behavior of a Hip resurfacing friction couple are rarely studied. G.S. Matharu (2015) observed as clinical outcomes, high rates of early failure of five hip resurfacing with a new composite ceramic (two-thirds polyurethane and one-third ceramic alumina) used for the acetabular component. Wen Zhang and al. (2010) investigated hip resurfacing lifetime predictions under static load, silicon nitride compared to alumina as ceramic materials, is indeed mechanically reliable and ideal for Hip resurfacing implant. This significant number of researches on the application of ceramics in Hip prosthesis whether total or resurfacing, unfortunately have not resulted in a definitive solution to avoid the risk of its fragility, so a proposal of another material is essential.

Many authors proposed the use of a composite material, P.Subhedar (2016) suggested the CF/PA12 composite as a more suitable material for Hip implant than metals and ceramics.

In this regard, this article aims to study the resistance of the Hip resurfacing prosthesis to applied dynamic loads and fatigue risk, using this new composite named CF/PA12, on a carbon fiber-based polymer (carbon fibers/polyamide12), whose biocompatibility had been demonstrated in the laboratory (S. Dimitrievska 2007; Dimitrievska et al. 2008). It presents a superior bioactivity in vitro and in vivo response to the hydroxyapatite coated composite femoral components (S.A. Hacking and al., 2010). Mechanical testing of femoral stems made from a carbon-fiber reinforced polymer composite were discussed in detail through M. Campbell, Bureau and Yahia (2008) study, the conclusion of this work shows that the bone-matching properties of this composite total hip prosthesis and its excellent fatigue resistance far exceeding the required fatigue resistance make CF / PA12 candidate material of choice for orthopedic appliances such as total hip prostheses. In addition, the application of a hip resurfacing cup made of carbon fiber/polyamide $12 \mathrm{CF} / \mathrm{PA} 12$ composite covered with a thin layer of cobalt chrome had the potential to reduce stress shielding, preserve bone stock and prevent from bone fracture compared to conventional metallic Hip resurfacing implants (Bougherara and Bureau, 2008). Vadean and Yahia (2007) proposed a comparison between Hip stems made of titanium alloy and CF/PA12 composite, stresses in composite stem are lower than those in Titan stem, and that the femoral bone implanted with composite structure sustains more load than the one implanted with Titane stem. Bougherara, Bureau and Yahia (2009) concluded from their study that CF/PA12 composite stem generates a better bone density pattern compared with the Titane based stem, indicating the effectiveness of the composite stem to reduce bone resorption caused by stressshielding phenomenon, this may result in an extended lifetime of total Hip arthroplasty. CF/PA12 composite stem might offer a better compromise between stress shielding and micromotions than the Titan stem with the same external geometry, these results obtained by Caouette, Yahia and 
Bureau (2011). Moreover, Bougheraraa and al. (2011) compared using finite element analysis. The mechanical behavior of the composite material of the total Hip arthroplasty stem with two standard commercially available metal hip implants. The study revealed that the Composite was less mechanically stiff, compared to the standard metallic hip stems. In another investigation, a new mechano-biochemical model, which is more comprehensive in the sense that it involves the coupling effect between the mechanical loading and bone biochemistry, was used to predict longterm bone density distribution around a CF/PA12 total Hip arthroplasty stem. The results were then compared to those obtained in femurs implanted with titanium alloy Titane and cobaltchrome-molybdenum implants. It was concluded that composite hip implant is more advantageous than metallic implant as it induces less stress shielding, develops a moderately dense trabecula at the vicinity of the implant and provides more uniform bone density (Pouria Tavakkoli Avval and al., 2011). We end with a research work on composite materials, which numerically evaluated an alternative method to cementing the stem to increase the stability of the femoral components of resurfacing implants, using the CF/PA12 composite material in the manufacture of an osseointegrated stem. This new implant with composite stem has therefore reduced the deviation of stresses compared to current metal implants (Caouette, 2012).

This research allows us to come back to certain points:

Cobalt chromium Hip resurfacing prostheses have been commercialized to date, and there are many improvements such as coatings, alloy diversity and design, but the potential problem of metal ions that is dangerous to human health remains always a disadvantage not to neglect.

The ceramic prosthesis is an interesting alternative to the metallic prosthesis and its resistance to corrosion and wear attracts attention, the disadvantage is that Ceramics fragility leads to an arthroplasty revision. We concluded that a long lifetime of a ceramic prosthesis is not at all obvious.

This new composite material (CF/PA12) has proven its biocompatibility in laboratories, its imitation to the human bone function that will allow a good prosthesis fixation, and its resistance to fatigue, all these positive characteristics led us to realize our study which is a static, dynamic and fatigue behavior finite element analysis of the hip resurfacing prosthesis designed entirely with this new composite material. The aim of this work is to confirm a long lifetime of the hip resurfacing prosthesis fully in CF / PA12.

\section{Materials and methods}

\subsection{Hip resurfacing model}

The proposed prosthesis is a Durom Hip resurfacing implant; with acetabular sizes which are 46 to $68 \mathrm{~mm}$ and femoral head sizes are 38 to $60 \mathrm{~mm}$. Our choice was the one with $46 \mathrm{~mm}$ of diameter; the acetabular implant was a cup with 54 of outside diameter, both of them distributed by the company Zimmer ${ }^{\circledR}$ (2012). We have chosen a relatively large diameter because the large diameter of the head approximates the anatomy and limits the risk of dislocation. The initial offset of the Hip prosthetic is preserved as well as the length of the neck, leaving the muscles with their initial tension. The bone capital is not only preserved by preserving the center of the head and the neck, but also by avoiding unnecessary alteration of the metaphyseal-diaphysis zone of the upper end of the femur (Kluess 2008). The length of the stem (L) is $L=D+3 \mathrm{~mm}, \mathrm{D}=$ diameter of the head, in our case equal to $46 \mathrm{~mm}$, therefore L equal to $49 \mathrm{~mm}$, (Zhang 2010).

The femoral neck diameter is $37 \mathrm{~mm}$ and that of the stem is $9 \mathrm{~mm}$ (Kluess 2008). The Durom cup was not completely hemispherical, it was a truncated hemisphere whose angle of opening was $165^{\circ}$, which allowed to increase the articular amplitudes and to respect as much as possible 
the acetabular bone stock; also the radial clearance must be superior or equal to $120 \mu \mathrm{m}$ (P.E. Ridon, 2016). The reduction of the radial clearance has a significant influence on the distribution of the contact pressure between the implant components (Bougherara 2008). Based on these outcomes, our diametral clearance prosthesis chosen was $130 \mu \mathrm{m}$. This clearance has one of the most influential characteristics of friction couples, which is defined as the difference between the internal diameter of the cup and the diameter of the head 2(R2-R1), which plays a major role in lubrication (Smith and Nephew 2011), all these dimensions are illustrated on our prosthesis model (Figure 1).

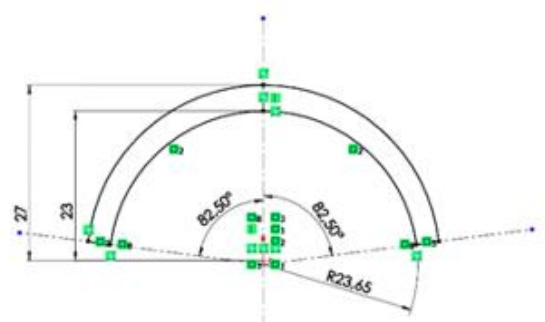

(a) Tow dimensional acetabular component skis.

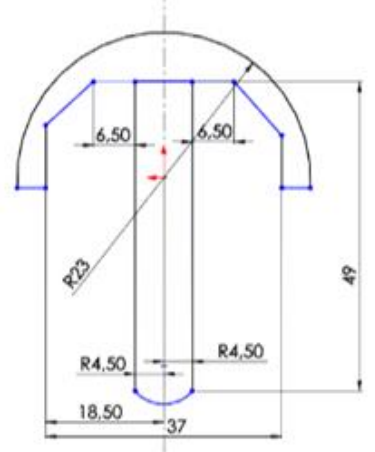

(c) Tow dimensional femoral head component skis.

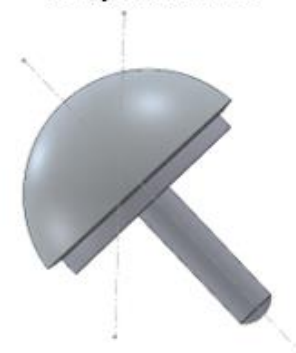

(e) Three-dimensional assembled prosthesis model.

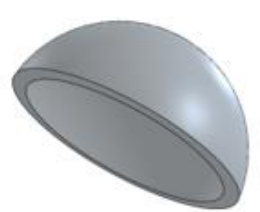

(b) Three dimensional acetabular component model.

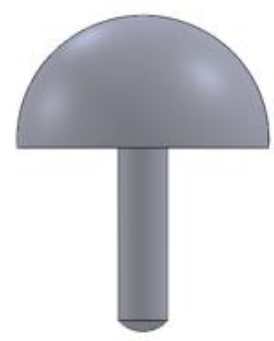

(d) Three-dimensional femoral head component model.

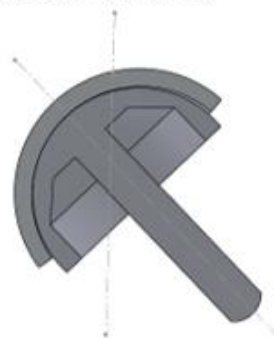

(f) Section illustrates the contact of prosthesis model.

Fig. 1. Geometry and model of CF/PA12 composite Hip resurfacing prothesis components.

The material used for the proposed hip resurfacing prosthesis design is CF/PA12 composite (carbon fiber composite with a polyamide 12 resin). With the increase in the number of layers of the laminate, the material is considered transverse isotropic (Caouette 2012), with six plies 
oriented at $\left({ }^{+} 45^{\circ}\right)$ represented with $\left[\left({ }^{+}-45^{\circ}\right)\right]_{6}$. Ply thickness considered is $0.5 \mathrm{~mm}$ (Bougheraraa and al. 2007) (Figure 2).

All mechanical composite proprieties are summarized in Table 1.

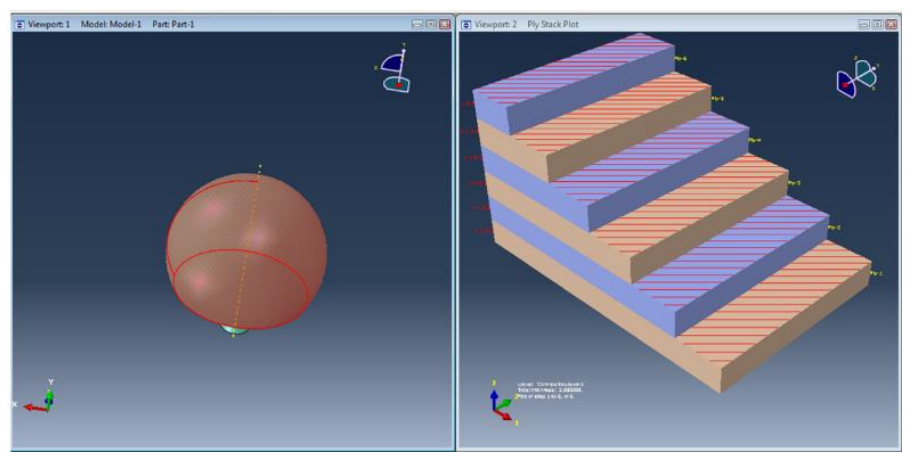

Fig. 2. Ply configurations used for CF/PA12 composite material $\left[\left({ }^{+}-45^{\circ}\right)\right]_{6}$

\begin{tabular}{|c|c|}
\hline properties & values \\
\hline $\begin{array}{c}E_{1}^{\text {compression }}, E_{2}^{\text {compression }} \\
\mathrm{E}_{2}^{\text {compression }}\end{array}$ & $10[\mathrm{GPa}]$ \\
\hline$v_{12}, v_{21} v_{12}, v_{21}$ & 0.4 \\
\hline $\mathrm{G}_{12}, \mathrm{G}_{21}$ & $4[\mathrm{GPa}]$ \\
\hline $\mathrm{E}_{3}$ & $15.7[\mathrm{GPa}]$ \\
\hline$v_{13} v_{23} v_{13}, v_{23}$ & 0.4 \\
\hline $\mathrm{G}_{13}, \mathrm{G}_{23}$ & $6[\mathrm{GPa}]$ \\
\hline Density (P.T. Avval. 2011) & $1.443\left[\mathrm{~g} / \mathrm{cm}^{3}\right]$ \\
\hline
\end{tabular}

Table 1. Mechanical composite proprieties (Caouette 2012).

\subsection{Loading conditions}

In this study, a load was applied to the surface of the bearing of the implant, the concept of "Pauwels balance" shows that the hip joint supports about 3 times the weight of the body. Therefore, for a person weighing $75 \mathrm{~kg}$, the force at the hip joint level can reach $2250 \mathrm{~N}$. In order to approach the reality, we consider a normal walking daily activity for our static analysis. Force components acting on the femoral head model allow us to reproduce all the phases of each movement by varying the Gait phase at different Gait cycle percentages of 2, 13, 19, 50 and 63\% (J.P. Hunga and al., 2004). In fatigue analysis, we are interested in the maximum values forces, $13 \%$ and $50 \%$ of the gait cycle are the positions when the hip contact forces are highest because it is an unipodal support (contact of only one foot on the floor) in the gait cycle, so $2 \%, 19 \%$ and $63 \%$ positions, hip contact forces are minimal because of the bipodal support (contact both feet on the floor) in the gait cycle (Bonnefoy-Mazure et al. 2015). Force components values corresponding to different positions of the Gait cycle are listed in (Table 2). These force components with respect to a vertical loading, were assumed to be positioned anatomically at an inclination angle of $45^{\circ}$ and an ante version of $0^{\circ}$ in the pelvic and femoral bone (Ahmet $\mathrm{C}$. Cilingir 2010), (Figure 3.a). Boundary conditions are applied by fixing the femoral stem component, since it is perfectly related to the surrounding bone. 
Modern software using the finite element method to solve contact problems usually approaches such problems via two basic theories that, although different in their approaches, lead to the desired solutions. One of the theories is known as the penalty function method, and the other as the Lagrange multipliers method. The main difference between them is the way they include in their formulation the potential energy of contacting surfaces.

The penalty function method, due to its economy, has received a wider acceptance. The method is very useful when solving frictional contact problems, while the Lagrange method, based on multipliers, is known for its accuracy. In our case we chose a penalty function method.

The contact at the bearing surfaces is considered with friction, using a friction coefficient of 0.25 (Griza and al. 2013), this composite material resists to wear, so it is not necessary to use a high friction coefficient, to be able to resist to wear, and allow a well lubricated condition (Figure 3.b).

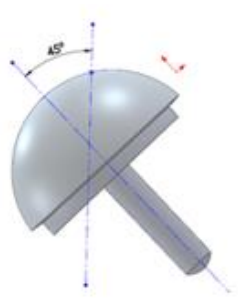

(a) Inclination angle of $45^{\circ}$ of the model.

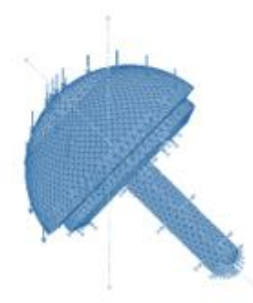

(b) Loading and boundary conditions prosthesis assembly.

Fig. 3. Positioning, loading and boundary conditions of the prosthesis entirely in CF / PA12 composite.

\begin{tabular}{|c|c|c|c|c|c|}
\hline \multirow{2}{*}{$\begin{array}{c}\text { Force } \\
\text { components }\end{array}$} & \multicolumn{5}{|c|}{ Percentage of Gait phase } \\
\cline { 2 - 6 } & $2 \%$ & $13 \%$ & $19 \%$ & $50 \%$ & $63 \%$ \\
\hline $\mathrm{F}_{\mathrm{x}}[\mathrm{N}]$ & -592.26 & -760.5 & -774 & -672.75 & -193.275 \\
\hline $\mathrm{F}_{\mathrm{y}}[\mathrm{N}]$ & 83.62 & -310.5 & -177.75 & 472.5 & 19.32 \\
\hline $\mathrm{F}_{\mathrm{z}}[\mathrm{N}]$ & -548.89 & -1532.25 & -1484.77 & -1568.25 & -596.25 \\
\hline
\end{tabular}

Table 2. Variation of static forces applied to the prosthesis according to the different positions of the Gait cycle during normal walking.

For dynamic analysis the climbing stairs activity leads to greater forces, of the order of 300 to $600 \%$ body weight (J.U. Perez, 2012), we choose the climbing stairs activity in our dynamic analysis, because the hip joint is more solicited. $600 \%$ of the body weight was considered as a dynamic force, The given function curve traced by the dynamic force was sinusoidal in shape in order to mimic human walking gait loading patterns on the hip joint (E. Rahim, 2010) as showed in (figure4). 


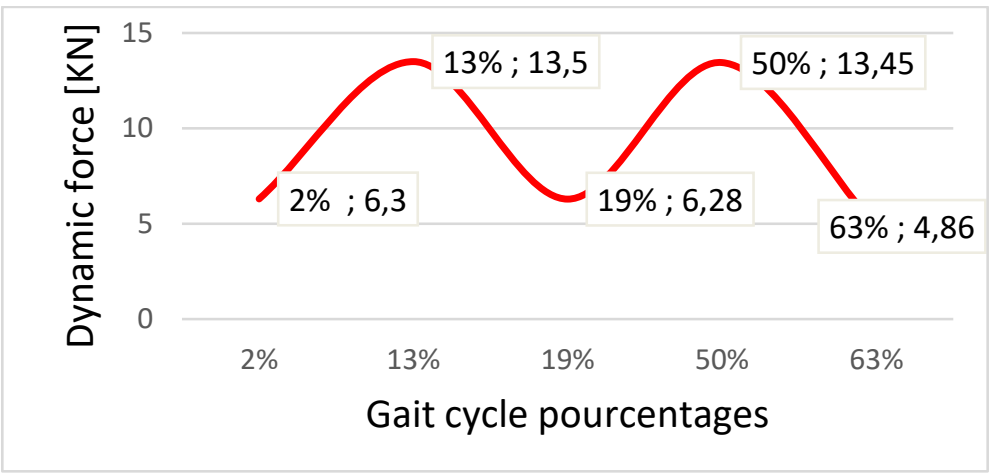

Fig. 4. Dynamic load application on CF / PA12 Hip resurfacing prosthesis for climbing stairs activity.

This dynamic analysis was carried out on CF/PA12 composite hip resurfacing prosthesis under repeated compression cyclic at a frequency of $6 \mathrm{~Hz}$, with the same positions of normal walking. The resulting forces components are shown below Table 3 .

\begin{tabular}{|c|c|c|c|c|c|}
\hline \multirow{2}{*}{$\begin{array}{c}\text { Force } \\
\text { components }\end{array}$} & \multicolumn{5}{|c|}{ Percentage of Gait phase } \\
\cline { 2 - 6 } & $2 \%$ & $13 \%$ & $19 \%$ & $50 \%$ & $63 \%$ \\
\hline $\mathrm{F}_{\mathrm{x}}[\mathrm{N}]$ & -3553.56 & -4563 & -4644 & -4036.5 & -1159.65 \\
\hline $\mathrm{F}_{\mathrm{y}}[\mathrm{N}]$ & 501.72 & -1863 & -1066.5 & 2835 & 115.92 \\
\hline $\mathrm{F}_{\mathrm{z}}[\mathrm{N}]$ & -3293.34 & -9193.5 & -8908.62 & -9409.5 & -3577.5 \\
\hline
\end{tabular}

Table 3. Variation of dynamic forces applied to the prosthesis according to the different positions of the Gait cycle during climbing stairs.

\subsection{Finite element analysis}

The finite element analysis was used to compute static, dynamic and fatigue behavior analysis on a hip resurfacing implant entirely made of CF/PA12 composite. This analysis is carried out using the SolidWorks software, compatible with the Abaqus finite element code on a DELL i5, $2.3 \mathrm{GHz}$ Intel (R) processor PC. Cup and femoral components were meshed using a higher order threedimensional element; SC6R: A 6-node triangular in-plane continuum shell wedge, generalpurpose continuum shell, reduced integration with hourglass control, finite membrane strains.

The average number of elements of the acetabular component model is 36407 and 39592 of the femoral head component. Contact elements were used between femoral head and acetabular components surfaces (Figure 5).

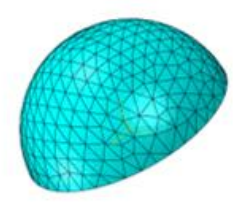

(a) meshed cup model

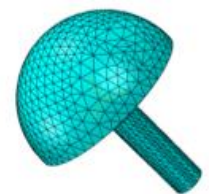

(b) meshed head model

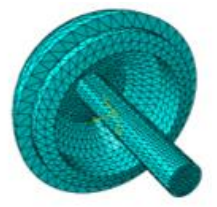

(c) meshed assembled implant model

Fig. 5. Finite element mesh of Hip prosthesis. 


\subsection{Fatigue analysis}

Dynamic cyclic loading simulation is a way to represent the effect of patient activity on the durability and design of the prosthesis; therefore, the effects of fatigue loads applied to the prosthesis were well illustrated by a dynamic analysis. In this study, the fatigue life of the prosthesis during stress analysis was based on the Goodman mean-stress fatigue theory, the mean stresses $\sigma \mathrm{m}$ and alternating stresses $\sigma \mathrm{a}$ in the Goodman fatigue life theory are defined by the relations below Eq. (1), Eq. (2) respectively. According to the modified Goodman theory the relation between mean and alternation stress is mentioned Eq. (3), where $S_{e}$ is the endurance limit and $\mathrm{S}_{\mathrm{ut}}$ is the ultimate tensile strength of the material. The fatigue factor of safety becomes as Eq. (4).

$$
\begin{gathered}
\sigma_{\mathrm{m}}=\frac{\sigma_{\max }+\sigma_{\min }}{2} \\
\sigma_{a}=\frac{\sigma_{\max }-\sigma_{\min }}{2} \\
\frac{\sigma_{\mathrm{a}}}{\mathrm{S}_{\mathrm{e}}}+\frac{\sigma_{\mathrm{m}}}{\mathrm{S}_{\mathrm{ut}}}=\frac{1}{\mathrm{n}} \\
n_{f}=\frac{1}{\frac{\sigma_{a}}{\mathrm{~S}_{e}}+\frac{\sigma_{m}}{\mathrm{~S}_{u t}}}
\end{gathered}
$$

\subsection{Results and discussion}

In this part, the results of the static, cyclic dynamic, and fatigue analysis were described and discussed.

\subsubsection{Static analysis results.}

The results obtained for the Von Mises stress analysis distribution in the implant was illustrated below (Table 4) and (Figure 6). We found that the highest Von Mises stress are at 13\%, 19\% and $50 \%$ positions (Figure 6). Maximum Von Mises stress values at different positions of the Gait cycle was mentioned below (Table 4).

\begin{tabular}{|c|c|c|c|c|c|}
\hline $\begin{array}{c}\text { Different positions of the } \\
\text { Gait cycle. }\end{array}$ & $2 \%$ & $13 \%$ & $19 \%$ & $50 \%$ & $63 \%$ \\
\hline $\begin{array}{c}\text { Maximum Von Mises } \\
\text { stress [MPA] }\end{array}$ & 4.841 & 10.630 & 10.040 & 10.056 & 3.798 \\
\hline
\end{tabular}

Table. 4. Static analysis maximum Von Mises stress values. 


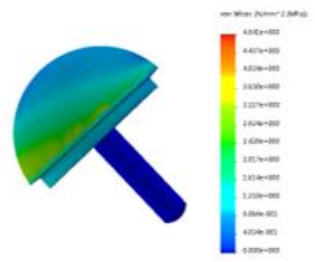

Von Mises stress at $2 \%$ position.

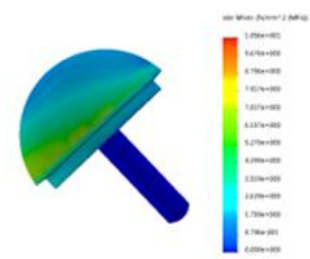

Von Mises stress at $50 \%$ position.

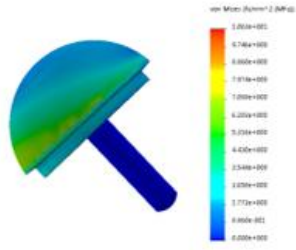

Von Mises stress at $13 \%$ position.

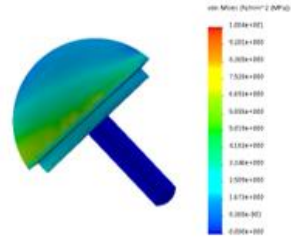

Von Mises stress at $19 \%$ position.

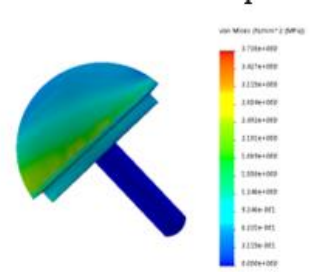

Von Mises stress at $63 \%$ position.

Fig. 6. The static analysis: Von Mises Stress distribution on the CF/PA12 hip resurfacing prosthesis under different levels of stress loading at different positions of the Gait cycle during normal walking.

\subsubsection{Static analysis discussions}

Highest Von Mises stress are at 13\%, 19\% and 50\% positions (Figure 6), due to the maximum force that will occur at the hip joint at the beginning of the stance phase of the Gait cycle, which is consistent with the literature. Noting that maximum Von Mises stress value 10.63 MPA at the most solicited position of the normal walking cycle is low and does not present any danger for the bone. This indicates that the design is safe using the new CF/PA12 composite material. The maximum of the simulated stresses in the prosthesis depends linearly on the force applied to the assembly, since the contact is continuous between the head and the cup; the contact force depends only on the materials properties and the applied force. We chose the $19 \%$ gait cycle position among the positions studied, to see the shape traced by the distribution of the contact surfaces stresses of the prosthesis (outside of the femoral head component and inside of the cup), due to the form which is almost the same in every position, the difference is in the size of this contact surface. The head and the cup being two compliant surfaces, the stresses obtained are distributed over approximately three quarters of the outer surface of the head and the inner surface of the cup as seen in the literature (J.U.Perez, 2012) (Figure $7 \mathrm{a}-\mathrm{b}$ ).

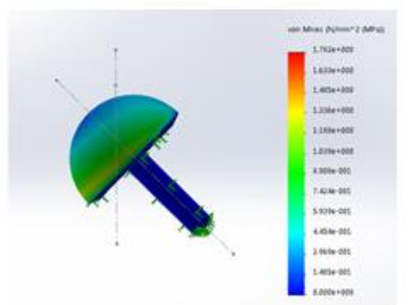

(a) Stresses distribution in the outer surface in the femoral head component

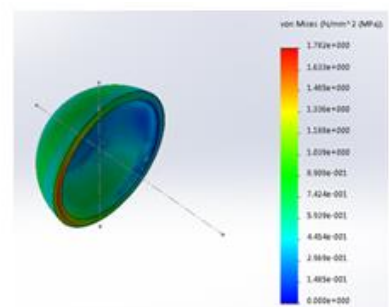

(b) Stresses distribution in inner surface of the cup.

Fig. 7. Stresses distribution in hip resurfacing components. 


\subsubsection{Dynamic analysis results}

The results of the dynamic cyclic analysis during climbing stairs showed the response of the hip joint to a dynamic load condition due to climbing stairs activity. In this analysis, stress concentration areas did not really change, despite the dynamic force applied on the new CF/PA12 composite hip resurfacing prosthesis even in $13 \%$ and $50 \%$ positions of Gait cycle when the implant is more solicited (Figure.8).

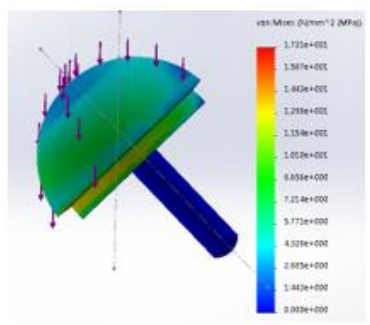

Max and $\min$ Von Mises stress at $2 \%$ position.

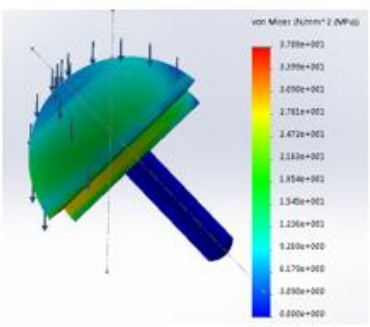

Max and min Von Mises stress at $13 \%$ position.

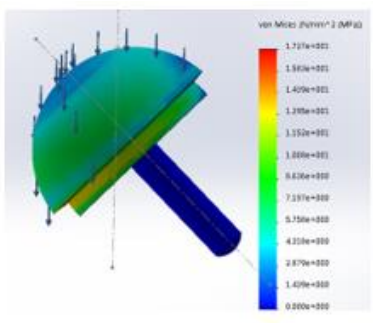

Max and min Von Mises stress at $19 \%$ position.

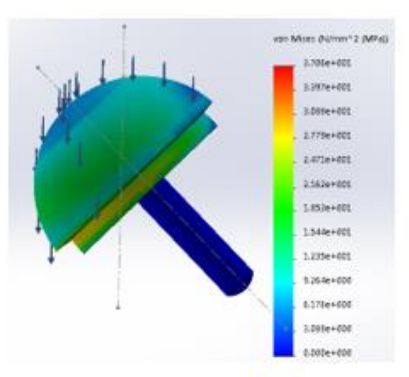

Max and min Von Mises stress at 50\% position

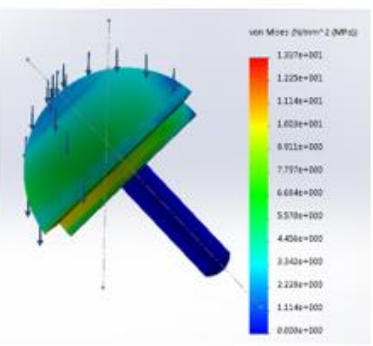

Max and min Von Mises stress at $63 \%$ position

Fig. 8. The dynamic cyclic analysis: Von Mises Stress distribution on the CF/PA12 Hip resurfacing prosthesis under different levels of stress loading at different positions of the Gait cycle during climbing stairs.

\subsubsection{Dynamic analysis discussions}

Two maximum Von Mises stress peaks of 37.08 and 37.06 MPA correspond respectively to $13 \%$ and $50 \%$ of the Gait cycle positions, are higher than those of the static analysis normal walking for the same positions, this is due to the magnitude of the force applied to the prosthesis during this activity. We confirm that the curve obtained represents two stress peaks at $13 \%$ and $50 \%$ positions of the Gait cycle similar to that seen in the literature (Hausselle 2007), which proves that our obtained results are reliable (Figure 9). 


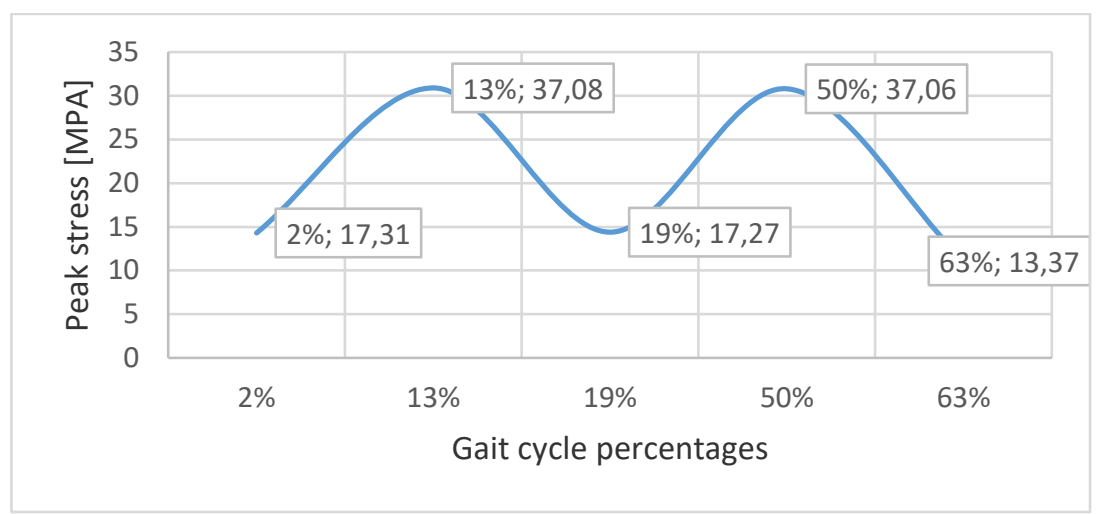

Fig. 9. Dynamic cyclic analysis distribution of maximum Von Mises stress in CF / PA12 Hip resurfacing prosthesis for climbing stairs activity.

Von Mises stress distribution in the Hip resurfacing prosthesis entirely made of CF/PA12 composite solicited by static and dynamic cyclic loads is shown below (Figure 10). From the comparison, we noticed that dynamic cyclic loading generates higher stresses compared to static loading. The peak stresses in the CF/PA12 hip resurfacing solicited by dynamic loads climbing stairs are the highest, around 37.08 MPA and 37.06 MPA at $13 \%$ and 50\% percentages of the Gait cycle, while for that solicited by static loads normal walking are of the order of 10.630 MPA and 10.056 MPA for the same positions respectively. For $13 \%$ and 50\% gait cycle positions in the static analysis they both represent the unipodal support of normal walking, and the forces corresponding to these two positions represent the two almost equal and highest values of the force applied on the hip, but the range between the value of the force corresponding to the unipodal support and the value of the force corresponding to the bipodal support in a normal walking is tight compared to this range in the climbing stairs because the peak force would be 23\% higher during climbing stairs than during normal walking (Perez 2012). Von Mises stress distribution results obtained under dynamic cyclic loads are 13.31 MPA, 17.27 MPA and 13.37 MPA correspond to $2 \%, 19 \%$ and $63 \%$ percentages of the Gait cycle respectively, we notice that these values are close because these three positions represent the bipodal support of the gait cycle. Despite the fact that the maximum Von Mises stresses are higher in the case of the dynamic cyclic load, they are still low compared with limit stress. Which is an advantage for the durability of the prosthesis and also for the bone. A low concentration of stress on the prosthesis will reduce the stress concentration generated by the implant on the bone, which reduces its risk of fracture.

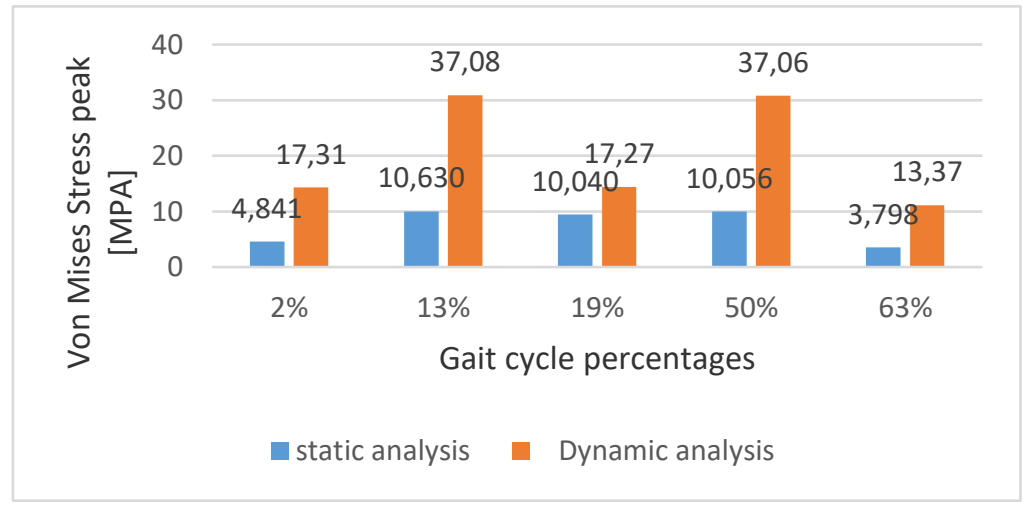

Fig. 10. Comparison of results of each position for each load approach analysis 


\subsubsection{Fatigue analysis results and discussions}

Von Misses stresses obtained from finite element analyses are utilized in fatigue life calculations. All fatigue analyses are performed according to infinite life criteria ( $\mathrm{N}=10^{9}$ cycles).

Static and dynamic cyclic analyses of the prosthesis should be conducted to ensure about long lifetime of the design. Prostheses are often designed according to the results of static analysis. Static finite element analyses are mostly conducted under body weight loads. However, dynamic effects may add up to about $10-20 \%$ or more loading to the prosthesis, which must be taken into account not to cause fracture or fatigue failure of the prosthesis. In our case of dynamic study, we consider climbing stairs activity with $600 \%$ of the body weight as a dynamic force. Based on static and dynamic cyclic analysis results of our resurfacing prosthesis designed entirely in CF / PA12 composite, conduct to predict that given low maximum stresses observed on our proposed prosthesis are far from the elastic limit (Figure 7-9), which is around 2500 to 3200 MPA. Whatever solicitations intensity that the prosthesis might undergo during daily human activities, it will never be exposed to failure. By synthesis two main works, that of Wen Zhang and al. (2010) who did a comparative finite element analysis showing lifetime predictions of alumina and silicon nitride as ceramic materials for the hip resurfacing prosthesis under the same boundary conditions and static loading. The lifetime predictions showed that silicon nitride is indeed mechanically reliable and ideal for hip resurfacing prostheses. With our study, we want to develop this resurfacing prosthesis by changing the ceramic material that is fragile with the CF / PA12 composite that resists to rupture by performing fatigue analysis under dynamic load (Figure 11). Based on the results mentioned above, our CF / PA12 prosthesis is very far from fatigue and we consider it as a better alternative to silicon nitride hip resurfacing prosthesis. Comparing our approach with the second study of Campbell, Bureau and Yahia (2008), their paper presents a thorough analysis of the mechanical properties and an evaluation of the performance of this innovative composite design for femoral stems made from a carbon-fiber reinforced polymer composite. Both short and long term mechanical testing of the hip stem were discussed in detail during their work. As a conclusion drawn from this work, the bone-matching properties of this composite-made total hip prosthesis and its excellent fatigue performance (Figure 11), by far surpassing required fatigue life make $\mathrm{CF} / \mathrm{PA} 12$ candidate material of choice for orthopedic devices such as total hip prostheses and might offer a long-term solution to stress shielding and bone desorption. The fatigue analysis behavior in their study is done on a total hip prosthesis stem, which gave us the idea to do a finite element fatigue analysis on a resurfacing prosthesis fully made (head and cup components) from this material. Our results confirm that the reliability of our prosthesis.CF/PA12 composite hip resurfacing prosthesis is really far from fatigue and failure risk. 


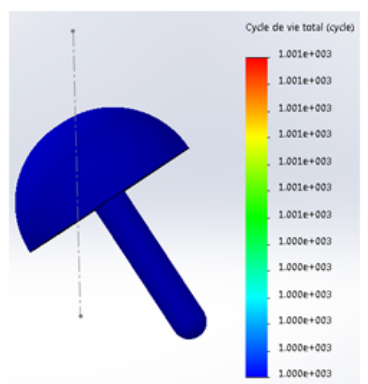

(a) Number of cycle life

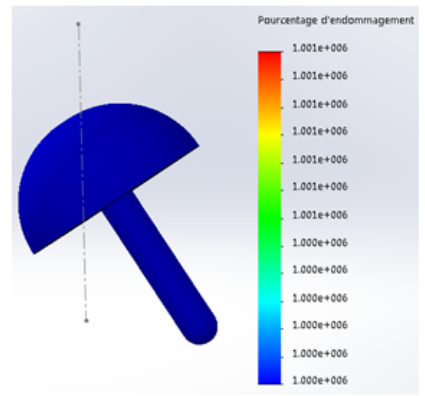

(b) Percentage of damage

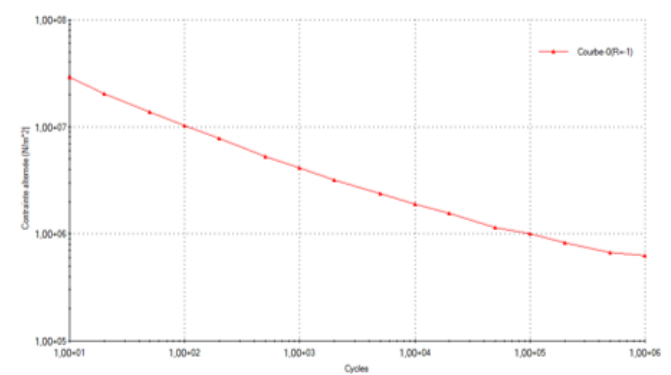

(c) S-N fatigue curve analysis

Fig. 11. Fatigue approach analysis

This plot (Figure 11), represents the percentage of the life of the structure consumed by the defined fatigue events $\left(\right.$ cycles $\left.=10^{7}\right)$.

\section{Conclusion}

This paper investigated static and dynamic behavior analysis of the proposed hip resurfacing prosthesis entirely designed with new CF/PA12 composite to conduct to determine the fatigue life of the prosthesis during stress analysis in case of walking and climbing stairs activity. We have focused our analysis on the couple composite / composite hip resurfacing prosthesis. Based on static and dynamic finite element analysis results, maximum Von Mises Stresses were calculated. The outcomes showed that maximum stresses on CF/PA12 composite prosthesis were low under static loading. The effects of the fatigue loads applied on the CF/PA12 composite hip resurfacing due to climbing stairs are well illustrated by dynamic cyclic analysis. So, under dynamic loading. The maximum stresses obtained were still low and so far from the yield stress, these indicate that the hip resurfacing prosthesis fully designed with CF/PA12 composite material is safe against fatigue under dynamic repetitive loadings. This fatigue performance of our hip resurfacing prosthesis entirely designed with CF/PA12 composite confirms that it is among the most appropriate implants for hip replacement. However, the finite element model needs to be improved, such as inclusion of surfaces conditions of the prosthesis components (roughness) would be expected to ensure better osseointegration.

The recommendations made following the work of this paper consist in optimizing the CF / PA12 hip resurfacing stem and cup geometric shape and their surface condition to improve osseointegration and stability of the implant. 
Acknowledgements The authors acknowledge support of the Research laboratory in production (LRP), University of Batna 2, Algeria.

\section{References}

Bonnefoy-Mazure A and Armand S (2015). Normal Gait, Orthopedic Management of Children with Cerebral Palsy,ISBN: 978-1-63483-318-9.

Cilingir AC (2010). Finite Element Analysis of the Contact Mechanics of Ceramic-on-Ceramic Hip Resurfacing Prostheses, Journal of Bionic Engineering 7, 244-253.

Pala B, Gupta S and Andrew MR (2010). Design considerations for ceramic resurfaced femoral head: effect of interface characteristics on failure mechanisms, Computer Methods in Biomechanics and Biomedical Engineering, Vol. 13, No. 2, 143-155.

Caouette $C$ (2012). design of a hip resurfacing prosthesis made of biomimetic composite material: development of digital assessment tools, doctoral thesis, Superior Technology School University Of Quebec, Canada.

Caouette C, Yahia LH, and Bureau MN (2011). Reduced stress shielding with limited micromotions using a carbon fiber composite biomimetic hip stem: a finite element model, Proc. IMechE Vol. 225 Part H: J. Engineering in Medicine.

Kluess D, Zietz C, Lindner T, Mittelmeier W, Schmitz PK and Bader R (2008). Limited range of motion of hip resurfacing arthroplasty due to unfavorable ratio of prosthetic head size and femoral neck diameter, Acta Orthopaedica, 79 (6): 748-754.

Rahim E (2010). Nondestructive Evaluation Of A Polymer Composite Hip Implant Using LockIn Thermography, Master thesis, Ryerson University, Ontario, Canada.

Matharu GS, Daniel J, Ziaee H, Mc Minn DJW (2015). Failure of a Novel Ceramic-on-Ceramic Hip Resurfacing Prosthesis, The Journal of Arthroplasty 30, 416-418.

Bougherara H, Bureau M, Campbell M, Vadean A, Yahia LH (2007). Design of a biomimetic polymer-composite hip prosthesis, Journal of Biomedical Materials Research Part A, Jul 82(1):27-40 in Wiley interscience.; DOI 10.1002/jbm.a.

Bougherara H, Bureau MN (2008). Biomimetic Composite-Metal Hip Resurfacing Implant, Hindawi Publishing Corporation Research Letters in Materials ScienceVolume, Article ID 368985.

Bougherara H, Bureau MN, Yahia LH (2009). Bone remodeling in a new biomimetic polymercomposite hip stem, Jan 92(1):164-74. in Wiley interscience.

Bougherara H, Zderoa P, Dubova A, Shaha S, Khurshida S, Schemitsch EH (2011). A preliminary biomechanical study of a novel carbon-fibre hip implant versus standard metallic hip implants, Medical Engineering \& Physics 33, 121-128.

Hunga JP, Chenb JH, Chianga HL, ShynWuc JS (2004). Computer simulation on fatigue behavior of cemented hip prostheses: a physiological model, Computer Methods and Programs in Biomedicine, 76, 103-113.

Hausselle J (2007). Study of the shock degradation of heads and cups of hip prostheses in bioceramic, doctoral thesis, Superior National School of Mines of Saint-Etienne, French, Order no. : 458 SGM.

Perez JU (2012). Multiscale Analysis of the durability of bioceramics for hip prostheses. In vitro and ex vivo studies, doctoral thesis, Superior National School of Mines of Saint-Etienne, French, NNT: 2012 EMSE 0642.

Campbell M, Bureau MN, Yahia LH (2008). Performance of CF/PA12 composite femoral stems, J Mater Sci: Mater Med 19:683-693.

Mencière ML (2014). Fracture of the modular cobalt-chromium femoral neckpiece of a total hip arthroplasty, Journal of Orthopedic and Traumatological Surgery 100, 419-422. 
Subhedar P, Thokal G, Patil CR (2016). Carbon fiber reinforced polyamide12 as a biomaterial for implant, International Journal of Current Engineering and Technology, E-ISSN 2277 4106, P-ISSN 2347 - 5161.

Ridon PE (2016). Comparative study of the metal-to-metal pair in hip resurfacing and in large diameter total prosthesis: analysis of the release of metal ions, doctoral thesis, Law and Health, University of Lille 2, French.

Avval PT, Samiezadeh S, Klika V, Bougherara H (2015). Investigating stress shielding spanned by biomimetic polymer-composite vs. metallic hip stem: A computational study using mechano-biochemical model, journal of the mechanical behavior of biomedical materials 41 , $56-67$.

Griza S, dos Santos SV, Ueki MM, Bertoni F, Strohaecker TR (2013). Case study and analysis of a fatigue failure in a THA stem, Engineering Failure Analysis 28, 166-175.

Hacking SA, Pauyo T, Lim L, Legoux JG, Bureau MN (2010). Tissue response to the components of a hydroxyapatite-coated composite femoral implant, Published online 31 March 2010 in Wiley InterScience.

Dimitrievska S, Petit A, Ajji A, Bureau MN, Yahia LH (2007). Biocompatibility of novel polymer-apatite nanocomposite fibers, published online 28 June 2007 in Wiley interscience.

Dimitrievska S, Whitfield J, Hacking SA, Bureau MN (2009). Novel carbon fiber composite for hip replacement with improved in vitro and in vivo osseointegration, October 2009, Journal of Biomedical Materials Research Part A 91(1):37-51, DOI: 10.1002/jbm.a.32175

Hamza S (2016). Compression Fatigue Crack Growth in Nacre and Its Implication on the Mechanical Performance of Orthopedic Implants, J Material Sci Eng 6:1.

Hamza S (2002). Study of the compression fatigue behaviour of bioceramics (Al2O3, ZrO2) used for the design of osteoarticular prostheses, doctoral thesis, University of Metz, French.

Smith and Nephew (2011). What is new in friction couples, metal / metal news, orthopedic master's degree, $\mathrm{N}^{\circ}$ 208, November 2011. Joint Commission 1213 T 86410, ISSN: 1148 2362.

Zhang W, Titze M, Cappi B, Wirtz DC, Telle R and Fischer H (2010). Improved mechanical long-term reliability of hip resurfacing prostheses by using silicon nitride, J Mater Sci: Mater Med 21:3049-3057.

Zimmer, Zimmer MMC resurfacing hip prosthesis - Operative Technique, 2012. 